\title{
Thermal and Microcanonical Rates of Unimolecular Reactions from an Energy Diffusion Theory Approach
}

\author{
Yin Guo and Donald L. Thompson \\ Department of Chemistry, Oklahoma State University, Stillwater, Oklahoma 74078-0444
}

\author{
William H. Miller* \\ Department of Chemistry, University of California, and Chemical Sciences Division, Lawrence Berkeley \\ Laboratory, Berkeley, California 94720
}

Received: May 18, 1999; In Final Form: September 13, 1999

\begin{abstract}
We present an energy diffusion theory approach for computing thermal and microcanonical unimolecular reaction rates by solving the general energy diffusion equation. The solution naturally provides the rates in the diffusion limit for fast reactions and the transition state theory (TST) limit for slow reactions. The reaction rates between the two limits can be easily obtained by solving a one-dimensional Schrödinger-like equation transformed from the diffusion equation. Employing a model system consisting of a set of harmonic oscillators interacting with a heat bath, the thermal rates from the low-temperature TST regime to the high-temperature diffusion regime are calculated to demonstrate their dependence on the size of the molecule. The approach also provides a practical means of obtaining microcanonical rates at considerable savings of computer time compared to trajectory simulations. The method is applied to the unimolecular dissociation of RDX (hexahydro1,3,5-trinitro-1,3,5-triazine), and its accuracy is demonstrated by comparison with the results from trajectory and Monte Carlo variational transition state theory calculations.
\end{abstract}

\section{Introduction}

Thermal unimolecular reactions are governed by competition between intermolecular and intramolecular processes. In the lowcollision-rate regime, collisional activation is the slower process and thus the rate limiting step. In the high-collision-rate regime, the intermolecular energy transfer is sufficient to maintain an equilibrium distribution of the activated complex, and the reaction can often be described by transition state theory (TST). In this work, we focus on the weak-collision regime where the average energy transfer per collision is small compared to the thermal energy. The weak-collision limit has been extensively studied. ${ }^{1-9}$ However, there is not a method available that naturally provides the rates regardless of slow or fast energy transfer.

We here present an energy diffusion theory treatment that covers the whole range from the weak-collision to the TST limit. The starting point is the widely used energy diffusion equation $^{4,5,8}$ which consists of two parts: the Fokker-Planck equation in energy space describing the collisional energy transfer, and a term accounting for chemical reaction. The solutions of the equation provide the rates over the entire range between the two limits. In the limit of fast collisional energy transfer, the equilibrium distribution is maintained and the rate is simply the TST value. In the limit of slow collisional energy transfer, energy activation is rate limiting and the equation gives the weak-collision-limit result. In the intermediate region, where the rate usually needs to be computed numerically, the energy diffusion equation is transformed into a one-dimensional Schrödinger-like equation to facilitate the solution.

Although the most direct applications of the energy diffusion theory are for thermal reactions, the theory can also be used to compute the microcanonical rates of large molecules. ${ }^{10-15}$ The basic idea is to consider the reaction coordinate as a subsystem that interacts with a bath consisting of the rest of the modes. The diffusion theory can then be applied in a straightforward way by taking the average energy per mode as the effective temperature. Generally speaking, at low energies where the reaction is slower than IVR (intramolecular vibrational energy redistribution) and thus rate limiting, statistical theories such as RRKM (Rice-Ramsperger-Kassel-Marcus) are accurate. At higher energies when the reaction becomes rapid and the rate of IVR is comparable to or even smaller than that of the reaction, the distribution near the transition state is depleted and hence the true dynamic rate deviates from statistical predictions. ${ }^{16}$ We have applied the energy diffusion theory to compute the microcanonical rates for large molecules in the high-energy (diffusion) regime by assuming that the IVR rate is much slower and thus determines the dynamic rate. ${ }^{11-14}$ The present approach enables us to obtain the reaction rates more generally for the entire range of energies from the statistical to the diffusion limit.

We first apply the method to compute the thermal rates for a model system of a set of harmonic oscillators embedded in a heat bath. The purpose is to demonstrate that the theory naturally provides the reaction rates from the weak-collision to the TST limit and also to investigate the dependence of the thermal rates on the number of degrees of freedom of the molecule. We then apply the method to calculate the microcanonical rates for the unimolecular dissociation of RDX. The accuracy of the method is demonstrated by comparison with classical trajectory and Monte Carlo variational transition state theory (MCVTST) results. 


\section{The Energy Diffusion Theory}

We consider a system coupled to a heat bath. Because of the stochastic nature of the collisions the evolution of the system is described by a Markovian master equation

$$
\begin{array}{r}
\frac{\partial P(E, t)}{\partial t}=\int_{0}^{\infty} \mathrm{d} E^{\prime}\left[K\left(E, E^{\prime}\right) P\left(E^{\prime}, t\right)-K\left(E^{\prime}, E\right) P(E, t)\right]- \\
k(E) P(E, t)
\end{array}
$$

where $P(E, t)$ is the energy probability function of the system, $k(E)$ is the microcanonical rate constant, and $K\left(E, E^{\prime}\right)$ is the transition kernel. If the collisional energy transfer is small compared to the thermal energy $k_{\mathrm{B}} T$, a Kramers-Moyal expansion of the master equation up to second order leads to a simpler energy diffusion equation ${ }^{5,6}$

$$
\frac{\partial P(E, t)}{\partial t}=\frac{1}{2} \frac{\partial}{\partial E}\left[D(E) P_{\mathrm{eq}}(E) \frac{\partial}{\partial E} \frac{P(E, t)}{P_{\mathrm{eq}}(E)}\right]-k(E) P(E, t)
$$

Here $P_{\text {eq }}(E)$ is the equilibrium distribution

$$
P_{\text {eq }}(E)=\rho(E) \mathrm{e}^{-E / k_{\mathrm{B}} T} / \int_{0}^{\infty} \mathrm{d} E \rho(E) \mathrm{e}^{-E / k_{\mathrm{B}} T}
$$

with $\rho(E)$ being the density of states, and $D(E)$ is the energy diffusion coefficient which is just the second moment of the transition kernel $K\left(E^{\prime}, E\right)$

$$
D(E)=\int \mathrm{d} E^{\prime} K\left(E^{\prime}, E\right)\left(E^{\prime}-E\right)^{2}
$$

Assuming impulsive collisions in configuration space and constant friction leads to ${ }^{8}$

$$
D(E)=\frac{k_{\mathrm{B}} T \xi}{\rho(E)} \int_{0}^{E} \mathrm{~d} E^{\prime} \rho\left(E^{\prime}\right)
$$

where $\xi$ is the friction constant and $\rho(E)$ the density of states of the system.

Equation 2 can be solved by expanding $P(E, t)$ as

$$
P(E, t)=\sum_{m} a_{m} \exp \left(-\mu_{m} t\right) \phi_{m}(E)
$$

where $\mu_{m}$ and $\phi_{m}(E)$ are the eigenvalues and eigenfunctions of the operator on the right-hand side of eq 2

$$
-\mu \phi(E, t)=\frac{1}{2} \frac{\partial}{\partial E}\left[D(E) P_{\mathrm{eq}}(E) \frac{\partial}{\partial E} \frac{\phi(E)}{P_{\mathrm{eq}}(E)}\right]-k(E) \phi(E)
$$

If the lowest eigenvalue $\mu_{0}$ is appreciably smaller than that of the first excited state, then at times $t \gg 1 / \mu_{1}$ the dominant contribution to $P(E, t)$ comes from the lowest eigenfunction, that is,

$$
P(E, t)=a_{0} \mathrm{e}^{-\mu_{0} t} \phi_{0}(E)
$$

In this case $P(E, t)$ is an exponential function in $t$, and $\mu_{0}$ is obviously the rate constant.

The first term on the right-hand side of eq 7 describes the energy supply from the bath to the system, and the second term accounts for the chemical reaction. These two competing factors determine the reaction rate. Analytical solutions of eq 7 in the two limits are readily available. In the limit of a slow reaction (small $k(E)$ ) where the chemical reaction is rate limiting, the reaction rate can be obtained using first order perturbation theory by taking the microcanonical rate $k(E)$ as the perturbation. The zeroth order lowest eigenvalue is zero and the corresponding eigenfunction is $P_{\mathrm{eq}}(E)$. The lowest eigenvalue through first order is then

$$
\mu=\frac{\int k(E) P_{\mathrm{eq}}(E) \mathrm{d} E}{\int P_{\mathrm{eq}}(E) \mathrm{d} E}
$$

which is simply the transition state theory expression as expected since in the case of a slow reaction the energy supply from the bath is sufficiently fast to maintain an equilibrium distribution of the system. In the limit of a fast reaction (large $k(E)$ ) where the energy supply is rate limiting, systems reaching the top of the barrier can be considered to react instantly. Thus it can be assumed that the probability function is zero for energies above the barrier and an analytical expression for the rate can be derived. ${ }^{4,7,8}$ Therefore, the solution of eq 7 naturally provides the rate constants for the entire region from slow to fast reaction limit.

However, eq 7 is usually not solvable analytically for the intermediate falloff region. To facilitate its numerical solutions, we define a new function $\chi(E)$

$$
\chi(E)=\left(\frac{D(E)}{P_{\mathrm{eq}}(E)}\right)^{1 / 2} \phi(E)
$$

to eliminate the first derivative term in the equation and transform it into a one-dimensional Schrödinger-like equation with a variable mass $1 / D(E)$

$$
\begin{aligned}
& \mu \chi(E)=-\frac{D(E)}{2} \chi^{\prime \prime}(E)+ \\
& \left\{k(E)+\frac{D(E)}{4}\left[\frac{\left(P_{\mathrm{eq}} D(E)\right)^{\prime \prime}}{P_{\mathrm{eq}} D(E)}-\frac{1}{2}\left(\frac{\left(P_{\mathrm{eq}} D(E)\right)^{\prime}}{P_{\mathrm{eq}} D(E)}\right)^{2}\right]\right\} \chi(E)
\end{aligned}
$$

For the sake of simplicity, in the study presented below we assume the system is harmonic. For a set of $n$ harmonic oscillators the density of states is

$$
\rho(E)=\frac{E^{n-1}}{(n-1) ! \prod_{i=1}^{n} h v_{i}}
$$

and the energy diffusion coefficient of eq 5 becomes

$$
D(E)=\frac{\xi E}{n \beta}
$$

with $\beta^{-1}=k_{\mathrm{B}} T$. Substituting eqs 12 and 13 into eq 7 yields

$$
\begin{aligned}
\mu \chi(E)=-\frac{\xi E}{2 n \beta} \chi^{\prime \prime}(E)+ & \\
& \left\{k(E)+\frac{\xi E}{4 n \beta}\left[\frac{\beta^{2}}{2}-\frac{\beta n}{E}+\frac{n(n-2)}{2 E^{2}}\right]\right\} \chi(E)
\end{aligned}
$$

Using the RRK (Rice-Ramsperger-Kassel) expression for $k(E)$

$$
k(E)=v\left(1-\frac{E_{\mathrm{b}}}{E}\right)^{n-1}
$$

with $E_{\mathrm{b}}$ being the barrier height, we solve eq 14 numerically to obtain the eigenvalues, and the lowest one is the rate constant. For $n>1$, we employ the standard discrete variable representation (DVR) method. For $n=1$, we do not obtain good convergence with DVR, thus we first derive the analytical 


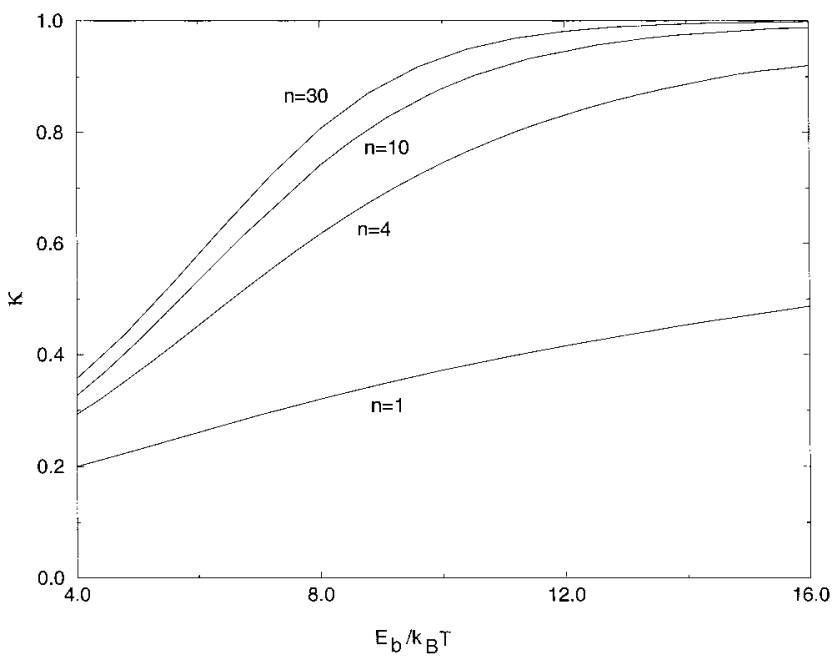

Figure 1. Scaled rate constants $\kappa=k / k_{\mathrm{TST}}$ versus $E_{\mathrm{b}} / k_{\mathrm{B}} T$ for a model system of $n$ harmonic oscillators. The results are for $n=1,4,10$, and 30. The parameters used are $v=0.1 \mathrm{fs}^{-1}$ and $\xi=0.01 \mathrm{fs}^{-1}$.

solutions of eq 14 without the $k(E)$ term and then use them as the basis functions to solve the full equation.

\section{Thermal Rate Constants}

Consider a model system that consists of a set of harmonic oscillators interacting with a heat bath. Using the RRK expression of eq 15 for the microcanonical rate $k(E)$, we have numerically solved eq 14, and the lowest eigenvalue is the rate constant. Figure 1 shows the scaled thermal rate $\kappa$ as a function of $\beta E_{\mathrm{b}}$, where $\kappa$ is defined as the ratio of the calculated rate to

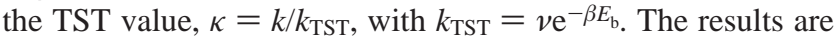
for $n=1,4,10$, and 30. The frequency factor was chosen as $v=0.1 \mathrm{fs}^{-1}$ and the friction constant $\xi=0.01 \mathrm{fs}^{-1}$. It is clear that the reaction is in the energy diffusion controlled regime at high temperatures and the TST regime at low temperatures. The transition to the TST regime moves to lower temperatures as the number of degrees of freedom of the subsystem decreases. However, the results for $n=10$ and 30 are not substantially different. Since the dependence of the rates on $v$ and $\xi$ are not sensitive to $n$ when it is large, the results indicate that in the transition region the thermal rates are not strongly dependent on the size of the molecule except for very small molecules with only a few degrees of freedom.

\section{Microcanonical Rate Constants}

We consider a molecule of $3 N-6$ modes that are separated into a reaction mode and $3 N-7$ bath modes. If $N$ is large and the energy $E$ in the reaction mode is much smaller than the total energy $E_{\text {total }}$, the equilibrium distribution of the reaction mode can be shown to be of the Boltzmann form ${ }^{10}$

$$
F_{\text {eq }}(E) \simeq \rho(E) \exp (-\beta E)
$$

where $\rho(E)$ is the density of states of the reaction mode and $\beta$ is the effective temperature corresponding to the average energy per mode

$$
\beta^{-1}=k_{\mathrm{B}} T=E_{\mathrm{total}} /(3 N-6)
$$

Thus, for a polyatomic molecule with a large number of degrees of freedom, if a reaction coordinate can be identified, then the rest of the vibrational modes can be considered as a bath and

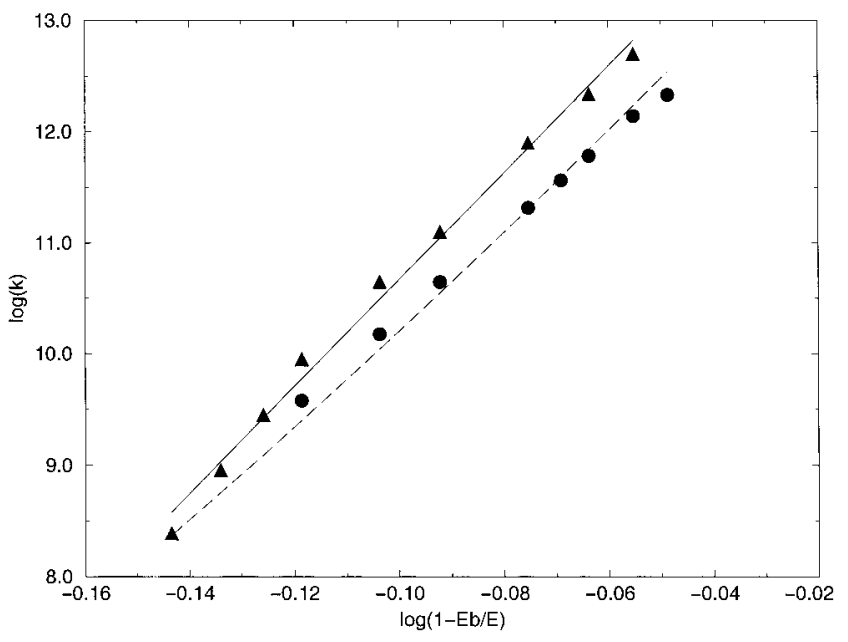

Figure 2. Microcanonical rate constants for the $\mathrm{N}-\mathrm{N}$ bond fission in RDX. The results are for the trajectory simulations (circles), the diffusion theory (dashed curve), Monte Carlo variational transition state theory (MCVTST) (triangle), and the RRK fit (solid line) to the MCVTST results. The MCVTST results are taken from ref 19 . The diffusion theory results were fitted at a total energy of $300 \mathrm{kcal} / \mathrm{mol}$ to the trajectory value (the fourth circle from the left) to obtain the friction constant $\xi$.

the reaction may be treated by the energy diffusion theory. Using the harmonic approximation for the reaction coordinate, the microcanonical rate can be obtained via eq 14 by substituting eq 17 for $\beta$ and letting the number of degrees of freedom of the subsystem $n$ be 1 .

For some simple reactions such as bond fissions, a reaction coordinate can be easily identified. For more complicated systems where there is not a simple reaction coordinate, one can use the reaction path Hamiltonian theory ${ }^{17}$ and take the intrinsic reaction coordinate as the subsystem and the normal coordinates orthogonal to the reaction path as the bath. Thus, the energy diffusion theory is readily applicable to general systems. Within the harmonic approximation for the reaction coordinate, the microcanonical rate can be obtained by solving eq 14 once the barrier height $E_{\mathrm{b}}$ and the friction coefficient $\xi$ are known. Assuming $\xi$ is independent of energy, we determine $\xi$ by fitting the rate constant calculated from eq 14 at a single energy to the corresponding term from a trajectory calculation. Then the rates at all energies can be obtained by solving eq 14 .

As a specific example, we have applied the method to the unimolecular dissociation of RDX. Trajectory simulations were also performed for comparison. The primary reaction channels are assumed to be a simple $\mathrm{N}-\mathrm{N}$ bond rupture and a concerted ring fission as illustrated in Figure 1 of ref 14. Chambers and Thompson ${ }^{18}$ have constructed a potential energy surface and performed classical trajectory simulations on it. Because of the availability of faster computers, we have recalculated trajectory results on the same potential energy surface using larger ensembles.

For the simple bond fission reaction in RDX, the $\mathrm{N}-\mathrm{N}$ bond length was chosen as the reaction coordinate and the rest of the modes as the bath. Figure 2 shows a comparison of the rate constants from the diffusion theory (dashed line), trajectory calculations (circles), and MCVTST (triangles). The solid line is a least-squares fit of the MCVTST rates to the RRK expression, eq 15, with fitting parameters $v=3.0 \mathrm{fs}^{-1}$ and $n=$ 47. The barrier $E_{\mathrm{b}}$ was chosen as the dissociation energy of the $\mathrm{N}-\mathrm{N}$ bond, $E_{\mathrm{b}}=47.8 \mathrm{kcal} / \mathrm{mol}$. The diffusion theory results were obtained by solving eq 14 with the RRK fitting parameter $v=3.0 \mathrm{fs}^{-1}$ used for the rate $k(E)$. The friction coefficient $\xi$ 


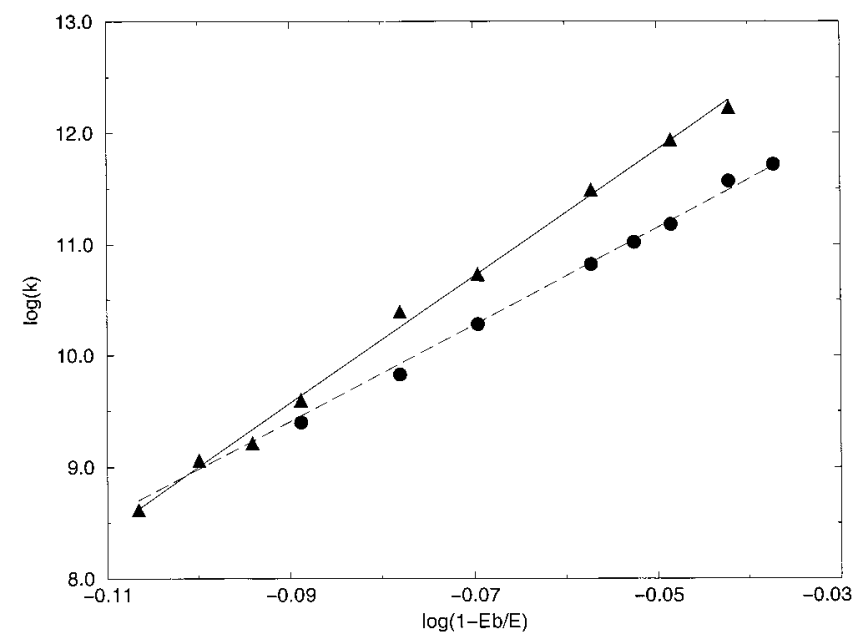

Figure 3. Same as Figure 2 except for the ring fission reaction in RDX.

was obtained by fitting the diffusion theory rate to the corresponding trajectory rate at $E_{\text {total }}=300 \mathrm{kcal} / \mathrm{mol}$ and then used to calculate the rates at other energies. At low energies, the dynamic rates converge to the statistical values. At high energies, the IVR becomes the slower and hence rate limiting step, and thus the dynamic rates deviate more from the corresponding statistical rates as the energy increases. It is clear that the diffusion theory provides an accurate description from the high energy dynamic regime down to the low energy statistical regime.

The ring fission reaction in RDX is a more interesting case and also provides a more stringent test of the theory. The RDX molecule breaks into three methylene nitramine $\left(\mathrm{CH}_{3} \mathrm{NNO}_{2}\right)$ molecules when three $\mathrm{C}-\mathrm{N}$ bonds in the ring rupture. There is no identifiable simple reaction coordinate for this reaction due to the highly coupled nature of the modes. For this kind of complicated system we use the idea of the intrinsic reaction coordinate (IRC) and take the IRC as the subsystem and the transverse vibrational modes as the bath. A plot of the potential energy along the minimum-energy path for the ring fission in RDX is given in Figure 2 of ref 18 with a barrier of $37 \mathrm{kcal} /$ mol. The ring fission reaction rates calculated by diffusion theory (dashed line), classical trajectories (circles), MCVTST ${ }^{19}$ (triangles), and the RRK fit ${ }^{19}$ to the MCVTST rates (solid line) are compared in Figure 3. The RRK fitting parameters are $v=$ $0.5 \mathrm{fs}^{-1}$ and $n=57$. The diffusion theory results were obtained by fitting to the trajectory result at $E_{\text {total }}=300 \mathrm{kcal} / \mathrm{mol}$ to determine the friction coefficient $\xi$. Again, diffusion theory gives good agreement with the statistical results at low energies and the dynamic results at higher energies.

\section{Summary}

We have presented an approach for obtaining thermal and microcanonical unimolecular reaction rates by solving the general energy diffusion equation. The basic assumption is that the energy exchange between the bath and the subsystem is diffusive and hence the evolution of the energy distribution of the subsystem can be described by the energy diffusion equation. The solution of the equation naturally covers the entire range from the weak-collision limit to the TST regime, and the rate constants can be easily obtained by solving a one-dimensional Schrödinger-like equation.

We have demonstrated the method by computing thermal rates for a model system in which the molecule is assumed to be a set of harmonic oscillators embedded in a heat bath. The results indicate that for large molecules the transition from the low-temperature TST regime to the high-temperature diffusion regime is not sensitive to the size of the molecule.

The main purpose of this work is to provide a practical means to compute the microcanonical rates for large molecules. The present approach enables us to extend our previous studies ${ }^{10-14}$ of IVR-controlled reactions to the more general case whether the rate of IVR is faster or slower than the rate of reaction. Assuming constant friction, the method predicts the rate constants for a wide range of energies by requiring only one rate from a trajectory calculation at a single energy to determine the friction coefficient. It thus provides a way of calculating microcanonical rates at considerable savings of computer time compared to the usual trajectory simulations. To test the accuracy of the method, we have applied it to the two reaction channels of the dissociation of RDX. In the simple $\mathrm{N}-\mathrm{N}$ bond rupture the breaking bond was chosen as the subsystem while in the more complicated ring fission reaction the intrinsic reaction coordinate was chosen as the subsystem. Comparisons with the trajectory and MCVTST results show that the method gives accurate rates from the low-energy statistical regime to the high-energy IVR-controlled region. Although the assumption of the energy diffusion theory-that the energy exchange between the bath and the reaction coordinate is diffusive-may not strictly hold, the calculated rate constants are nevertheless quite accurate, suggesting that the present semiempirical method provides a practical means of obtaining the microcanonical rates for large molecules.

Acknowledgment. This work was supported by the U.S. Army Research Office Grant No. DAAG55-98-1-0089 (Y.G. and D.L.T.) and by the Director, Office of Sciences, Office of Basic Energy Sciences, Chemical Sciences Division of the U.S. Department of Energy under Contract No. DE-AC03-76SF00098, by the Laboratory Directed Research and Development (LDRD) project from National Energy Research Scientific Computing (NERSC) Center, Lawrence Berkeley National Laboratory, and also by National Science Foundation Grant CHE 97-3258 (W.H.M.).

\section{References and Notes}

(1) Kramers, H. A. Physica 1940, 7, 284.

(2) Hynes, J. T. In Theory of Chemical Reaction Dynamics; Baer, M., Ed.; CRC: Boca Raton, FL, 1985; Vol. IV, p 171. 251.

(3) Hanggi, P.; Talkner, P.; Borkovec, M. Rev. Mod. Phys. 1990, 62,

(4) Nikitin, E. E. Theory of Elementary Atomic and Molecular Processes in Gases; Clarendon: Oxford, U.K., 1974.

(5) Keck, J.; Carrier, G. J. Chem. Phys. 1965, 43, 284.

(6) Hanggi, P.; Thomas, H. Phys. Rep. 1982, 88, 207.

(7) Troe, J. J. Chem. Phys. 1977, 66, 4745.

(8) Borkovec, M.; Berne, B. J. Chem. Phys. 1985, 82, 794

(9) Zawadzki, A. G.; Hynes, J. T. Chem. Phys. Lett. 1985, 113, 476

(10) Shalashilin, D. V.; Thompson, D. L. J. Chem. Phys. 1996, 105, 1833.

(11) Shalashilin, D. V.; Thompson, D. L. J. Chem. Phys. 1997, 107, 6204.

(12) Shalashilin, D. V.; Thompson, D. L. In Highly Excited States: Relaxation, Reactions, and Structures; Mullin, A., Schatz, G. C., Eds.; American Chemical Society: Washington, DC, 1997; p 81.

(13) Guo, Y.; Shalashilin, D. V.; Krouse, J. A.; Thompson, D. L. J. Chem. Phys. 1999, 110, 5514.

(14) Guo, Y.; Shalashilin, D. V.; Krouse, J. A.; Thompson, D. L. J. Chem. Phys. 1999, 110, 5521.

(15) Kato, T. J. Chem. Phys. 1998, 108, 6611.

(16) IVR-limited reactions have been previously discussed; see, for example, ref 10 and references therein.

(17) Miller, W. H.; Handy, N. C.; Adams, J. E. J. Chem. Phys. 1980, $72,99$.

(18) Chambers, C. C.; Thompson, D. L. J. Phys. Chem. 1995, 99, 15881. 961.

(19) Shalashilin, D. V.; Thompson, D. L. J. Phys. Chem. A 1997, 101 